\title{
CA-AODV: Congestion Adaptive AODV Routing Protocol for Streaming Video in Mobile Ad Hoc Networks
}

\author{
Boraiah RAMESH $^{1}$, Dhanabalachandran MANJULA ${ }^{2}$ \\ ${ }^{I}$ Department of Computer Science and Engineering, College of Engineering, Anna University, Chennai, India \\ ${ }^{2}$ Department of Computer Science and Engineering, College of Engineering, Anna University, Chennai, India \\ Email: sanchara@gmail.com ${ }^{1}$, manju@annauniv.edu ${ }^{2}$ \\ Received July 20, 2008; revised October 10, 2008; accepted October 14, 2008
}

\begin{abstract}
Whenever streaming of multimedia based data such as video, audio and text is performed traffic will be more and network becomes congested in mobile ad hoc networks. The present routing protocols are not able to cope up with this situation. It is observed that network congestion is the dominant reason for packet loss, longer delay and delay jitter in streaming video. Most of the present routing protocols are not designed to adapt to congestion control.

We propose a new routing protocol, Congestion Adaptive AODV Routing Protocol (CA-AODV), to address the congestion issues considering delay, packet loss and routing overhead. To evaluate their performance, we have considered mpeg4 for streaming video data using network simulator (NS2). CAAODV outperforms present protocols in delivery ratio and delay, while introducing less routing protocol overhead. The result demonstrates that integrating congestion adaptive mechanisms with AODV is a promising way to improve performance for heavy traffic load in multimedia based mobile ad hoc networks.
\end{abstract}

Keywords: AODV, Streaming, Congestion, Multimedia, Video

\section{Introduction}

A mobile ad hoc network (MANET) is a collection of mobile nodes that form a wireless network without the use of a fixed infrastructure i.e., base stations or access points or any centralized administration. Ad hoc wireless networks are self-creating, self-organizing, and selfadministering. They come into existence solely by interacting among their constituent mobile nodes and only such interactions are used to provide the necessary control and administration functions supporting such networks. The ad hoc wireless networks offer unique benefits and versatility for certain environments and certain applications. The preexisting fixed infrastructure and base stations are not being prerequisite to such networks. They can be created and used any time, anywhere. Such networks could be intrinsically faultresilient, for they do not operate under the limitations of a fixed topology. Indeed, since all nodes are allowed to be mobile, the composition of such networks is necessarily time varying. Addition and deletion of nodes occur only by interactions with other nodes, no other agency is involved. Such perceived advantages elicited immediate interest in the early days among military, police, and rescue agencies in the use of such networks, especially under disorganized or hostile environments like isolated scenes of natural disaster and armed conflict. In recent days, home or small office networking and collaborative computing with laptop computers in a small area (e.g., a conference or classroom, single building, convention center) have emerged as other major areas of potential application. In addition, people also recognize that ad hoc networking has obvious potential application in all the traditional areas of interest for mobile computing.

Streaming multimedia type of data is very challenging issue in mobile ad hoc networks. Many researchers have considered these factors very seriously and are working in this direction $[1,2]$. Thus, our aim is to develop a routing protocol that provides alternate non congested path if node become congested. The congested node will immediately provide congestion status to concerned node in order to take necessary action. Providing congestion status is desirable for many applications, as this allows them to alter the data they transmit. For example, several 
visual compression techniques, such as MPEG-4 [3] and H. 263 [4], are designed to meet various channel conditions. Without this congestion status information, the node may not be alert to change its path, causing congestion in the network and a large number of dropped packets.

In mobile ad hoc networks, a message sent by a mobile node may be received simultaneously by all of its neighboring nodes. Messages directed to mobile nodes not within the sender's transmission range must be forwarded by neighbors, which thus act as routers. Due to mobility it is not possible to establish fixed paths for message delivery through the network. Mobile Ad hoc networks are composed of mobile stations communicating solely through wireless links [5]. Routing protocols are classified as proactive or reactive, depending on whether they keep routes continuously updated, or whether they react on demand.

The routing protocols [6] can also be categorized based on congestion-adaptive versus congestion-un adaptive routing. The congestion unawareness in routing in MANETs may lead to the following issues.

1) Maximum delay to find a new route: Traditional routing protocol takes maximum time for detecting congestion using a suitable control mechanism. In severe congestion situations, it may be better to use a new route. The problem with an on-demand routing protocol is the delay it takes to search for the new route.

2) Huge routing overhead: In case a new route is needed, it takes processing and communication effort to discover it. If multi-path routing is used, though an alternate route is readily found, it takes effort to maintain multiple paths.

3) Heavy packet loss: Many packets may have already been lost by the time congestion is detected. A typical congestion control solution will try to reduce the traffic load, either by decreasing the sending rate at the sender or dropping packets at the intermediate nodes or doing both. The consequence is a high packet loss rate or a small throughput at the receiver.

The above problems become more visible in largescale transmission of traffic intensive data such as multimedia data. In such situation congestion is more probable and the negative impact of packet loss on the service quality is more of significance. We have proposed Congestion Adaptive AODV Protocol which tries to prevent congestion from occurring in the first place and be adaptive should a congestion occur. The ns2 simulation results show that the proposed protocol significantly improves the packet loss rate and end-toend delay while enjoying small protocol overhead and high-energy efficiency as compared to AODV [7], DSDV [8], DSR [9], and TORA [10]. Our proposed Adaptive Congestion AODV protocol tries to prevent congestion from occurring in the first place and it is adaptive to network congestion.
The remainder of the paper is organized as follows: Related Works is presented in Section 2. The proposed congestion adaptive routing protocol is presented in Section 3. In Section 4 we investigate simulation results and analysis of obtained results. Finally, Section 5 concludes the paper.

\section{Related Works}

Since Mobile ad network nodes are highly dynamic in nature, congestion is a main factor for more packet loss and longer delay. Traditional AODV is not an effective method under this situation in ad hoc networks [11]. It doesn't take any necessary precautions to handle the nodes which become congested under heavy network traffic. The modified version of AODV called CADV favors nodes with short queuing delays by adding it into the route to the destination. While this modification may improve the route quality, the issues of long delay and high overhead when a new route needs to be discovered remain unsolved. Furthermore, CADV is not congestion adaptive. It offers no remedy when an existing route becomes heavily congested. A similar routing protocol has been proposed in [12], Dynamic Load Aware Routing protocol, which favors low routing load in the routing path during route discovery. CADV and DLAR are both are single path on-demand routing protocols. Some of the multi path protocols were suggested in [1315] which are extensions of AODV and DSR.

AODV (Ad hoc On-demand Distance Vector) [7] is a dynamic, self-starting, multi-hop on-demand routing protocol for mobile wireless ad hoc networks. AODV discovers paths without source routing and maintains table instance of route cache. This is loop free and uses destination sequence numbers. AODV also maintains active routes only while they are in use and delete the stale (unused) route. AODV performs Route Discovery using control messages Route Request (RREQ) and Route Reply (RREP) whenever a node wishes to send packet to destination. The source node in network broadcasts RREQs to neighbors and uses an expanding ring search technique. The forward path sets up in intermediate nodes in its routing table with a lifetime association using RREP. When route is broken, destination or intermediate node moves RERR to the source node. When RERR is received, source node reinitiate discovery is still needed.

DSR (Dynamic Source Routing) [9] is reactive, simple and efficient routing protocol for multi-hop wireless ad hoc networks of mobile nodes. DSR uses source routing and this protocol is composed of two main mechanisms: Route Discovery and Route Maintenance. Both the mechanisms work together entirely, on-loopfree routing. They can rapidly discover the changes in the network routes and are designed for mobile ad hoc networks of up to about two hundred nodes and they work well even with high rates of mobility. The source 
route is needed when some nodes originate a new packet destined for some node by searching its route cache or initiating route discovery using RREQ and RREP messages. On detecting the break, DSR sends RERR message to source for new route.

The Destination-Sequenced Distance-Vector (DSDV) [8] Routing Algorithm is based on the idea of the classical Bellman-Ford Routing Algorithm with certain improvements. Every mobile station maintains a routing table that lists all available destinations, the number of hops to reach the destination and the sequence number assigned by the destination node. The sequence number is used to distinguish stale routes from new ones and thus avoid the formation of loops. The stations periodically transmit their routing tables to their immediate neighbors. A station also transmits its routing table if a significant change has occurred in its table from the last update sent. So, the update is both time-driven and event-driven.

The routing table updates can be sent in two ways: a "full dump" or an incremental update. A full dump sends the full routing table to the neighbors and could span many packets whereas in an incremental update only those entries from the routing table are sent that has a metric change since the last update and it must fit in a packet. If there is space in the incremental update packet then those entries whose sequence number has changed will be included into it. When the network is relatively stable, incremental updates are sent to avoid extra traffic and full dump are relatively infrequent. In a fastchanging network, incremental packets can grow big so full dumps will be more frequent.

The Temporally-Ordered Routing Algorithm (TORA) [10] is "an adaptive routing protocol for multi-hop networks". TORA is a distributed algorithm so that routers only need to maintain knowledge about their neighbors. TORA also maintains states on a per destination basis like other distance-vector algorithms. It uses a mix of reactive and proactive routing. Sources initiate route requests in a reactive mode. At the same time, selected destinations may start proactive operations to build traditional routing tables. Usually, routes to these destinations may be consistently or frequently required, such as routes to gateways or servers. TORA supports multiple path routing. It is said that TORA minimizes the communication overhead associated with adapting to network topology changes. The reason is that TORA keeps multiple paths and it does not need to discover a new route when the network topology changes unless all routes in the local route cache fail. Hence, the trade off is that since multiple paths are used, routes may not always be the shortest ones.

TORA uses the concept of height associated with a certain destination to describe the routing metric used by routers. Like water flows in pipes, routers with higher heights may forward packet flows to neighbors with lower heights. Note that since heights for routers are associated with particular destinations, the paths to forward packets are also associated with the corre- sponding destinations. In networks using TORA, an independent copy of TORA runs for each possible destination. So for different destinations, routers may have different heights and links can have different directions.

\section{CA-AODV: Congestion Adaptive Routing Protocol}

The proposed routing protocol is designed to ensure the availability of primary route as well as alternative routes and reduce the route overhead. If congestion happens at any point of time between source and destination nodes on primary route, concerned node warns its previous node about congestion. The previous node uses a non congested alternate route to the destination node. Since video data is very sensitive in delay and packet loss, the measurement of congestion has been considered here depending on average packet delivery time and packet delivery ratio. The Congestion adaptive AODV is reactive routing protocol and has the following three divisions.

- Congestion status setup

- Route Discovery Process

- Route Maintenance Process

\subsection{Congestion Status Setup}

Calculate time taken at every intermediate node from source node periodically. The calculated time at intermediate node is called as calculated delay $\mathrm{C}_{\mathrm{d}}$. The average delivery time should be calculated by source node i.e. expected delay $\left(E_{d}\right)$ to reach destination. Check $C_{d}$ with expected delay $E_{d}$ in the following manner and set up value of $\mathrm{C}_{\mathrm{s}}$ congestion status.

The status of congestion can be indicated by three levels: Forward, Alert and Drop. The Forward level means that packet can be forwarded to the next node, Alert means continue with remaining packets, but not for a longer time, and Drop level means there is no alternate way to forward packet, just drop it.

- If calculated time $\mathrm{C}_{\mathrm{d}}<\mathrm{E}_{\mathrm{d}}$, the value of $\mathrm{C}_{\mathrm{s}}$ will be in Forward level.

- If calculated time $\mathrm{C}_{\mathrm{d}}<=\mathrm{E}_{\mathrm{d}}$, the value of $\mathrm{C}_{\mathrm{s}}$ will be in Alert level.

- If calculated time $\mathrm{C}_{\mathrm{d}}>\mathrm{E}_{\mathrm{d}}$, the value of $\mathrm{C}_{\mathrm{s}}$ will be in Drop level.

\subsection{Route Discovery Process}

During the route-request phase, each node which receives a RREQ packet will determine level of congestion status. If $\mathrm{Cs}$ is in forward level, RREQ packet will be forwarded to next neighbor node. If $\mathrm{C}_{\mathrm{s}}$ is in alert level, RREQ packet might be forwarded but this is not continued for a longer time. If Cs is in drop level, RREQ packet will be dropped. 
We have modified conventional AODV as per our requirements. For all the nodes on the main route, RREQ packet will be forwarded to next node. The destination node will send the RREP packet back to the source as the conventional AODV does and then complete the route discovery process.

The data structures used are as follows:

The main routing table is represented by $\operatorname{MRT}\left[\mathrm{S}_{\mathrm{n}}, \mathrm{D}_{\mathrm{n}}\right]$. It specifies the entry for destination $D_{n}$ in the routing table of node $S_{n}$. The MRT $\left[S_{n}, D_{n}\right]$.attr specify the value for the attribute attr. The traffic can be reduced by dropping RREQ packets when congestion status is "Drop" and also stop broadcasting RREQ packets.

1) The main routing table metric attribute is set to 1 : $\operatorname{MRT}\left[\mathrm{S}_{\mathrm{n}}, \mathrm{D}_{\mathrm{n}}\right]$. nc_metric $=1$, for $\mathrm{n}$ nodes. Set destination node and its congestion status level: $\operatorname{MRT}\left[\mathrm{S}_{\mathrm{n}}\right.$, $\left.\mathrm{D}_{\mathrm{n}}\right]$.nc_hop $=\mathrm{D}$ and MRT $\left[\mathrm{S}_{\mathrm{n}}, \mathrm{D}_{\mathrm{n}}\right]$.hop_status= "Forward". Set main table as not congested node: $\operatorname{MRT}\left[S_{n}, D_{n}\right] . n c \_h o p=-1$ for every other node.

2) Whenever node $S_{n}$ receives an updated packet from its neighbor node $S_{\text {next }}$, it will check if MRT[ $S_{\text {next }}$, $\left.D_{n}\right]$.nc_status="Drop" and MRT $\left[S_{\text {next }}, D_{n}\right]$.nc_status ="Alert" then node $S_{n}$ will initiate non congested path discovery process towards the node $S_{\text {next }}$ obtained from the update packet.

3) Searching for non congested optimal route: Set TTL to $2 \mathrm{x} \mathrm{k}$ in non congested request packets, where $\mathrm{k}$ is distance between node $S_{n}$ and non-congested node $S_{i}$ on the main route.

4) If non congested node is already present in main routing table, drop non congested request packet.

5) If timeout occurs after certain period, delete the entries in the non-congested alternate table.

6) The traffic splitting can be done effectively as follows: If next main node MRT $\left[S_{n}, D_{n}\right]$.hop = "drop" the incoming packets will follow main Link.

$S_{n} \rightarrow \operatorname{MRT}\left[S_{n}, D_{n}\right]$.hop and with probability $p=$ MRT $\left[\mathrm{S}_{\mathrm{n}}, \mathrm{D}_{\mathrm{n}}\right]$. prob $=0.5$.

Non congested link $S_{n} \rightarrow \operatorname{MRT}\left[S_{n}, D_{n}\right] . n c \_h o p$ will have equal chance $(1-\mathrm{p}=0.5)$.

\subsection{Route Maintenance Process}

The route maintenance of our proposed modified version of AODV algorithm will take necessary actions compared to traditional AODV. If there is a broken route detected by monitoring error message RERR and/or node does not receive any reply message from a specific neighbor within a predefined interval of time, it remarks that routes as invalid and sends an error message to the upstream nodes. Once the error message has been received by previous node, it would select best alternate route.

\section{System Simulation Design}

We have implemented proposed protocol using Network
Simulator NS-2 [16] version 2.28. We have compared CA-AODV to DSR, AODV, DSDV and TORA, the most popular MANET routing protocols. In following sections observations are discussed.

\subsection{System Simulation model}

We built a simulation model to support video transmission which consists of 100 mobile nodes to form ad hoc network within the $1500 \mathrm{~m} \times 800 \mathrm{~m}$ rectangular field. The nodes were equipped with omni-directional antennas. To test the performance of our proposed routing protocol with other protocols in MANET environment we have used MPEG4 video traffic generator [17]. The packet size used in our simulations is 512 bytes and the raw channel bandwidth is 2 Mbps. The ten pairs of source destination flows are randomly chosen to observe congestion. The routing buffer at the network layer could store up to 128 data packets. The MAC layer was based on IEEE 802.11 CSMA and interface queue at MAC layer could hold 50 packets. The random waypoint model [18] was used with maximum node speed of $4 \mathrm{~m} / \mathrm{s}$ as suggested in [19].

The simulations were run for 900 seconds for ten different simulations with different pause times, where a higher pause time reflects lower mobility, 0 indicates a high mobility scenario, while a pause time of 900 is considered a stable network. For each connection, the source generated 512-byte data packets at a constant bit rate $(\mathrm{CBR})$ in the traffic model.

\subsection{Parameters Monitored}

We have evaluated the performance of CA-AODV by considering three important parameters: Data packet delivery ratio, Normalized routing overhead and End-toend data packet delay [20].

Data packet delivery ratio: The ratio of the number of packets sent by the source nodes to the number of packets received by the destination nodes.

Normalized routing overhead ratio: The ratio of the total number of routing packets transmitted to the number of data packets delivered. For packets sent over multiple hops, each transmission of a packet over a hop counts as one transmission. Protocols that generate large amounts of routing overhead increase the probability of packet collision and data packet delays in network interface queues.

End-to-end data packet delay: The delay in transmitting data packets through wireless links plus the delay in the network interface queues due to network congestion. This metrics includes all the possible delays caused by buffering during the route discovery latency, queuing at the interface queue, retransmission delays at the MAC layer.

\subsection{Simulation Result Analysis}


The performance of proposed CA-AODV was evaluated by comparing it with AODV, DSR, DSDV and TORA. We considered various numbers of sessions with different packet rates and mobility models.

Figures 1 to 3 show the performance of CA-AODV and other routing protocols with different packet rates (20 packets/sec, 30 packets/sec and 40 packets/sec), and a pause time of 500s. The proposed routing protocol provide a high packet delivery ratio, small normalized routing overhead and low end-to-end delay than AODV and other routing protocols. As a consequence, AODV has slightly same performance compare to CA-AODV under light traffic - this is also observed at 10 packets/sec. This is because of less traffic load. When the packet rate increases to 20, CA-AODV performs better than AODV and other routing protocols, and this becomes more pronounced as the traffic load increases. At 20 packets/s, the packet delivery ratio of CA-AODV outperforms AODV by $12 \%$, DSR by $17 \%$ and DSDV by $20 \%$ and TORA by $22 \%$. Whenever the packet rate is increased, CA-AODV uses less routing overhead (up to 20\% less) than AODV. The end-to-end delay is slightly more than AODV because of some alternate routes that are not the shortest.

Thus with CA-AODV the traffic load is more balanced, and the probability of packet loss is reduced. Furthermore, in congested nodes, alternate routes should be selected, resulting in a significant increase in packet delivery, decrease in routing overhead and increase in delays.

Different mobility models were simulated by using different pause times. It was observed that mobility has a great impact on the performance of CA-AODV and other routing protocols. Performance is always worse with high mobility, but mobility has a slightly greater impact on CA-AODV than AODV and other routing protocols. Figures 4 to 6 show the performance of CA-AODV and other protocols with $20 \mathrm{CBR}$ sessions. The CA-AODV has a lower packet delivery ratio than AODV by $2 \%$ at 0s pause time (high mobility), and the routing overhead is $10 \%$ less than with AODV. At 900 s pause time (low mobility), CA-AODV has a 5\% to10\% higher packet delivery ratio than AODV and other protocols, and requires $30 \%$ to $40 \%$ less routing overhead than AODV and other protocols.

The delay variation is less than that of AODV and DSR which makes our protocol more suitable for multimedia kind of applications as shown in Figures 2 and 5 .

The routing overhead incurred by CA-AODV is very less when compared to other routing protocols. This is shown in Figure 3 and 6 . When packet rate was 50 packets per second the proposed protocol incurred less routing overhead and delivered $21.34 \%$ more data than AODV. This is because, upon link breakage, AODV tried to establish a new route to the destination by broadcasting RREQ and RREP packets, CA-AODV protocol tried to make use of non congested available route and uses route request packets very often. The overhead to maintain non-congested paths in proposed algorithm is kept small by minimizing the use of multiple paths.

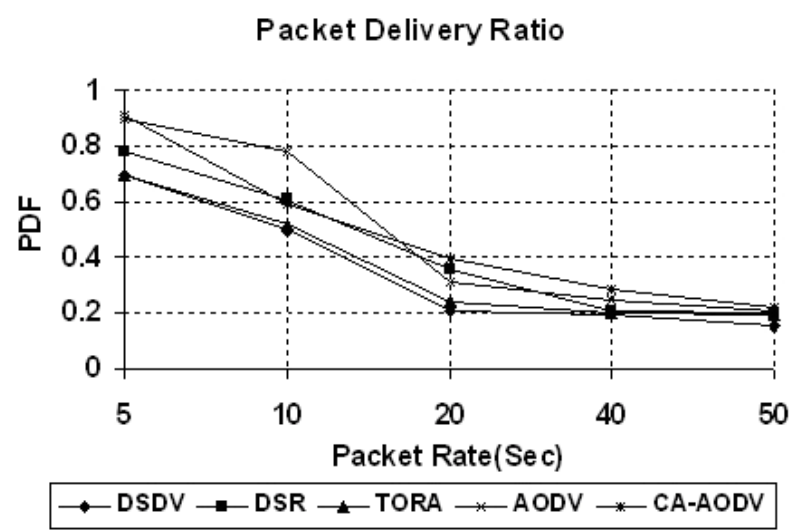

Figure 1. Comparison of packet delivery ratio vs. packet rate.

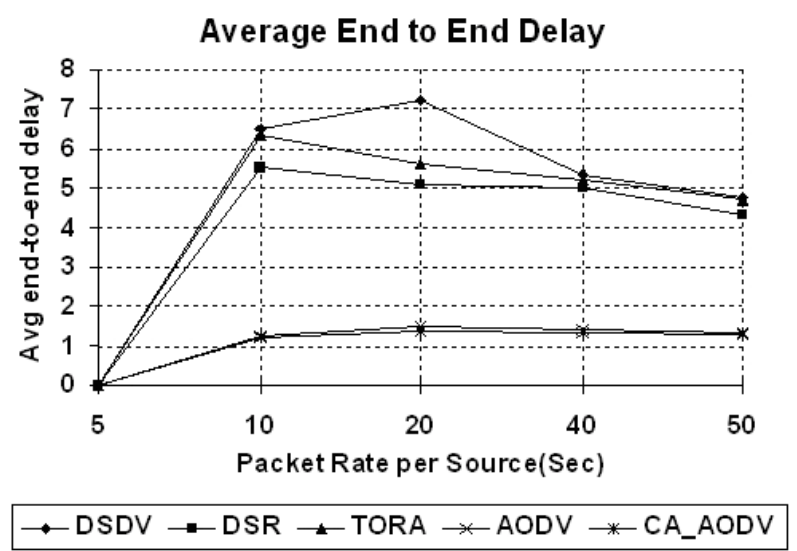

Figure 2. Comparison of average end to end delay vs. packet rate.

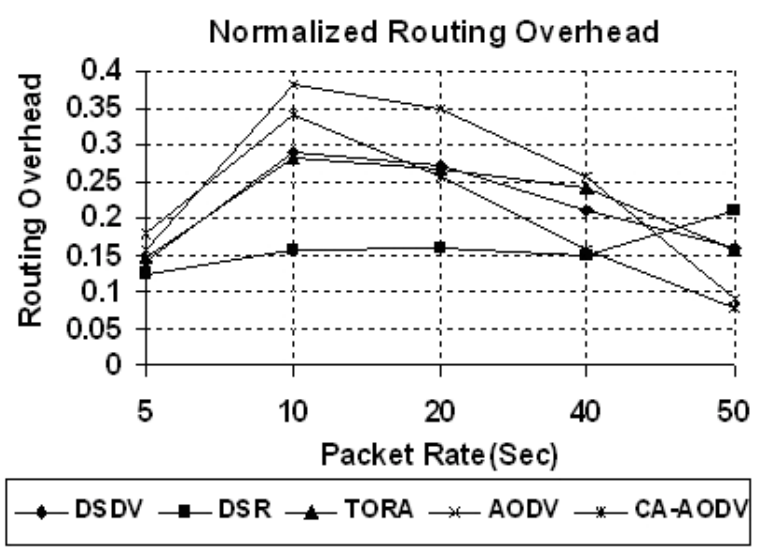

Figure 3. Comparison of normalized routing overhead vs. packet rate. 


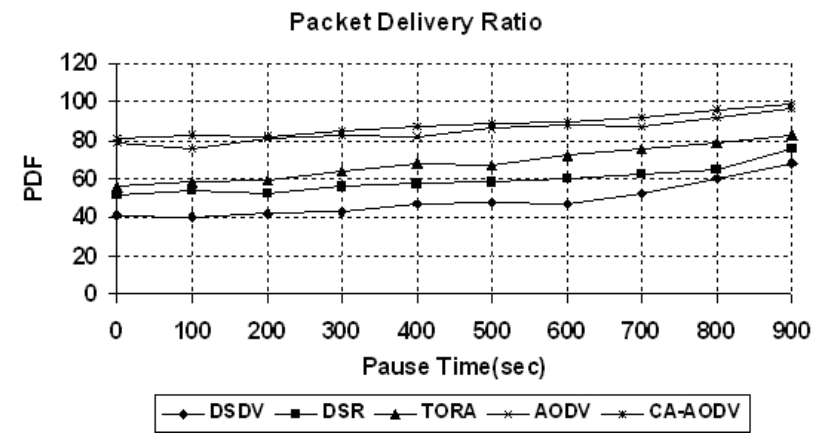

Figure 4. Comparison of packet delivery ratio vs. different pause time.

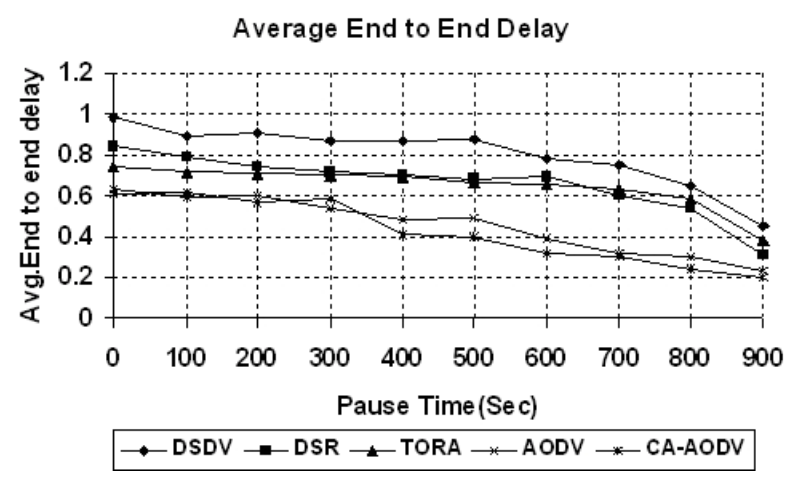

Figure 5. Comparison of average end to end delay vs. different pause time.

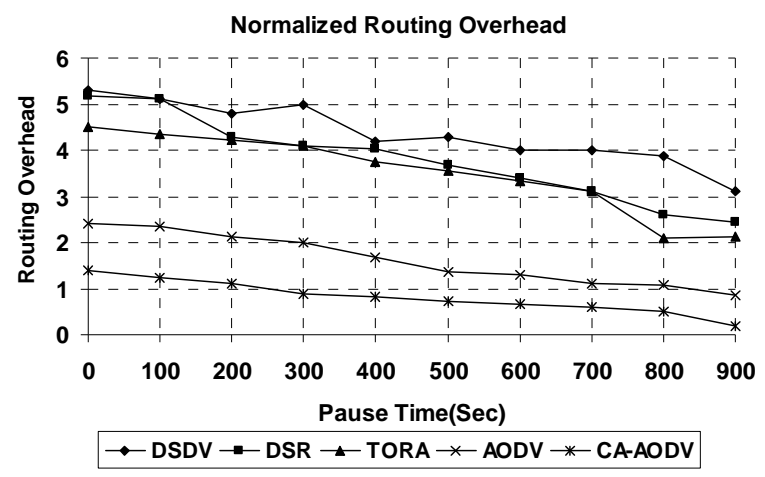

Figure 6. Comparison of Normalized Routing Overhead vs. different Pause time.

\section{Conclusions}

In this paper, we have proposed a new method to adapt to network congestion for video streaming in mobile ad hoc networks. The existing MANET protocols are not adaptive to network congestion and cannot handle the heavy traffic load while offering services to multimedia applications. The proposed Congestion Adaptive AODV Routing Protocol (CA-AODV) reduces packet losses than other routing protocols in real time transmission. The non-congested alternate route concept in the proposed method help next node that may go congested.
Whenever a node becomes aware of congestion ahead, it finds a non-congested alternate route that will be used to avoid congestion that is about to happen. The part of incoming traffic is split and then sent on the noncongested route, making the traffic coming to the congested node less. Thus congestion can be avoided. Proposed Algorithm does not incur heavy overhead due to maintenance of non-congested alternate paths. It also offers high packet delivery when the traffic in heavy. The delay incurred while establishing new connection is low because of using existing non-congested paths. Thus the proposed algorithm in mobile ad hoc networks is especially designed for multimedia applications. The difference between CA-AODV and other protocols were examined. Results were presented considering various situations to show the effectiveness of our proposed method. It is clear that CA-AODV can provide good performance comparable to AODV and other protocols in light traffic, and better performance in heavy traffic at a cost of slight longer delays.

\section{References}

[1] C. H. Ke, C. H. Lin, C. K. Shieh, and W. S. Hwang. "A novel realistic simulation tool for video transmission over wireless network," IEEE (SUTC2006), June 5-7, 2006.

[2] A. Lo, G. Heijenk, and I. Niemegeers, "Performance evaluation of MPEG-4 video streaming over UMTS networks using an integrated tool environment," International Conference, Netherlands, 2005.

[3] Y. Zhang, "Very low bit rate video coding standards," in Proceedings of Visual Communication and Image Processing, Vol. 2501, May 1995, pp. 1016-1023. 572 IEEE Journal on Selected Areas in Communications, Vol. 23, No. 3, March 2005.

[4] G. Coete, B. Erol, M. Gallant, and F. Kossentini, "H.263+: Video coding at low bit rates," IEEE Transactions on Circuits Systems for Video Technology, Vol. 8, No. 7, pp. 849-866, November 1998.

[5] G. S. Lauer, "Packet-radio routing, in: Routing in communications networks", Editor: M. E. Steenstrup (Rentice-Hall, 1995), Chapter 1, 35, pp. 1-396.

[6] S. Ramanathan, M. E. Steenstrup, "A survey of routing techniques for mobile communications networks, mobile networks and applications," Vol. 1, pp. 98-104, 1996.

[7] C. E. Perkins, "Ad hoc on demand distance vector (AODV) routing," IEFT Internet Draft, November 1998, http://www.ieft.org/internetdrafts/draft-ietf-manet-aodv02.txt.

[8] C. E. Perkins and P. Bhagwat, "Highly dynamic destination sequenced distance vector routing (DSDV) for mobile computers," Proceedings of ACM SIGCOMM' 94, London, UK, pp. 234-244, AugustSeptember 1994.

[9] D. B. Johnson, D. A. Maltz, and J. Broch, "Dynamic source routing for multihop wireless ad hoc networks," in Ad Hoc Networking, edited by Charles E. Perkins, Addison-Wesley, Chapter 5, pp 139-172, 2001. 
[10] V. Park and S. Corson, "Temporally-ordered routing algorithm (TORA) Version 1 functional specification," IETF Internet Draft, work in progress, draft-ietf-manettora-spec-04.txt, 2001.

[11] Y. Lu, W. Wang, Y. Zhong, and B. Bhargava, "Study of distance vector routing protocols for mobile ad hoc networks," Proceedings IEEE International Conference Pervasive Computing and Comm. (PerCom), pp. 187-194, March 2003.

[12] S. J. Lee and M. Gerla, "Dynamic load-aware routing in ad hoc networks," Proceedings IEEE International Conference on Communications, pp. 3206-3210, June 2001.

[13] S. J. Lee and M. Gerla, "Split multipath routing with maximally disjoint paths in ad hoc networks," Proceedings IEEE International Conference on Communications, pp. 3201-3205, June 2001.

[14] M. Marina and S. Das, "On-demand multipath distance vector routing in ad hoc networks," Proceedings IEEE International Conference Network Protocols (ICNP), pp. 14-23, 2001.

[15] Nasipuri and S. R. Das, "On-demand multipath routing for mobile ad hoc networks," Proceedings IEEE
International Conference on Computer Communications and Networks (IC3N), October 1999.

[16] NS-2. Network Simulator. http://www.isi.edu/nsnam/ns/.

[17] M. Chowdhury, Y. Tu, and T. Pham, "MPEG traffic picture matrices via TCP over ATM," Proceedings of the International Conference on Computer Science, Software Engineering, Information Technology, e-Business, and Applications (CSITeA'03), Rio de Janeiro, Brazil, pp. 128-132, June 5-7, 2003.

[18] A. Boukerche, "Performance evaluation of routing protocols for ad hoc wireless networks. Mobile networks and applications," Vol. 9, pp. 333-342, 2004.

[19] Y. Lu, W. Wang,Y. Zhong, and B. Bhargava. "Study of distance vector routing protocols for mobile ad hoc networks," in IEEE International Conference on Communication, Helsinki, Finland, pp. 3201-3205, June 2001.

[20] C. Perkins, E. M. Royer, S. R. Das, and M. Marina, "Performance comparison of two on-demand routing protocols for ad hoc networks," IEEE Personal Communications Magazine, Vol. 8, No. 1, pp. 16-28, February 2001. 